\title{
MIELOLIPOMA EM GLÂNDULA ADRENAL DE UM CANINO
}

\author{
MYELOLIPOMA IN THE ADRENAL GLAND OF A CANINE
}

Monique Togni ${ }^{1}$, Joana Cristina Smaha de Jesus Lima ${ }^{2}$, Maisa Martins Quirilos Assis ${ }^{3}$, Willian Fontini Marangon ${ }^{3}$, Jean Carlos dos Santos da Luz ${ }^{4}$

\footnotetext{
${ }^{1}$ Universidade Regional Integrada do Alto Uruguai e das Missões, Santiago, RS, Brasil.

2 Universidade Federal do Paraná, Palotina, PR, Brasil.

${ }^{3}$ Centro Universitário Integrado de Campo Mourão, Campo Mourão, PR, Brasil.

${ }^{4}$ Universidade de São Paulo, São Paulo, SP, Brasil.

*Autor de correspondência: moniquetognimartins@gmail.com
}

Recebido: 10 de junho de 2020; Aceito: 29 de setembro de 2020.

\section{RESUMO}

O presente relato visa descrever os achados macroscópicos e histopatológicos de um canino com mielolipoma em glândula adrenal esquerda. Tumores de glândula adrenal são neoplasias frequentes em cães, porém mielolipomas são geralmente encontrados em bovinos ou primatas sendo infrequente em outras espécies. Um cadáver de um canino, sem raça definida, com diagnóstico clínico de doença renal aguda foi encaminhado ao Laboratório de Patologia para a realização da necropsia. Na abertura da cavidade abdominal foi encontrada uma massa crânio-medial ao rim esquerdo que circundava o hilo renal. $O$ rim esquerdo apresentava leve aumento de volume. A massa foi diagnosticada microscopicamente como Mielolipoma e no rim esquerdo foram encontradas alterações que sugeriam isquemia renal. $O$ diagnóstico de mielolipoma de adrenal foi considerado um achado incidental de necropsia, sendo a causa da morte deste canino uma doença renal bilateral de origem não determinada. Porém, apesar deste tumor não estar relacionado ao óbito do animal lesões compatíveis com isquemia no rim esquerdo foram encontradas, demonstrando, que tumores benignos de adrenal, como o mielolipoma, podem gerar lesões por compressão no rim.

Palavras-chave: Canino. Endócrino. Neoplasia. Rim.

\section{ABSTRACT}

The present report aims to describe the macroscopic and histopathological findings of a canine with myelolipoma in the left adrenal gland. Although the adrenal gland tumors are frequently found in dogs, myelolipomas are generally found in cattle or primates and uncommon in other species. A corpse of a mixedbreed canine, with a clinical diagnosis of acute kidney disease, was referred to the Pathology Laboratory for necropsy. At the opening of the abdominal cavity, a vascular mass medial to the left edge surrounding the renal hilum was detected. The left kidney had a higher volume. The mass was microscopically diagnosed as myelolipoma, and changes were found in the left kidney, suggesting renal ischemia. The diagnosis of adrenal myelolipoma was considered an incidental 
necropsy finding, which was the cause of the death of this canine, while the origin of the bilateral kidney disease was not provided. Although this tumor was not directly related to the death of the animal compatible with ischemia in the left border, benign adrenal tumors, such as myelolipoma, could generate compression at the border.

Keywords: Canine, Endocrine, Neoplasia, Kidney.

\section{INTRODUÇÃO}

Neoplasmas em glândulas adrenais são frequentes em cães (LABELLE et al., 2004) e estes podem ser classificados em tumores de córtex adrenal, sendo eles o adenoma, carcinoma e mielolipoma e tumores medulares de adrenal como o feocromocitoma, neuroblastoma e ganglioneuroma.

Entretanto, os mielolipomas são encontrados comumente em adrenais de bovinos ou primatas, sendo este tumor infrequente em outras espécies (CAPEN, 2002), sendo poucas vezes relatada em cães (LABELLE; COCK, 2005; TURSI et al., 2005; MORANDI et al., 2007; FRANÇA; ECCO; GUEDES., 2008; LEDUR et al., 2012).

O mielolipoma é considerado um tumor benigno, geralmente inativo, composto por adipócito bem diferenciados e diferentes células hematopoiéticas, sendo geralmente considerado um achado incidental de necropsia (CAPEN, 2002; PATEL et al., 2006; ECCO; LANGOHR, 2016), porém pode apresentar alterações compressiva em órgãos adjacentes (CAPEN, 2002; PATEL et al., 2006).

Este trabalho tem por finalidade descrever os achados macroscópicos e histopatológicos de um cão uma neoplasia incomum em adrenal esquerda.

\section{RELATO DO CASO}

No Centro Veterinário do Centro Universitário Integrado de Campo Mourão foi atendida um canino, fêmea, adulto, sem raça definida (SRD), apresentando episódios frequentes de vômitos e apatia. Nos exames laboratoriais o animal apresentou elevação de creatinina $(5,07 \mathrm{mg} / \mathrm{dL})$, ureia $(165,8 \mathrm{mg} / \mathrm{dL})$ e fosfatase alcalina $(179,8 \mathrm{U} / \mathrm{L})$, sugerindo clinicamente doença renal.

O animal foi internado e após dois dias morreu sendo realizada a necropsia. O cadáver apresentava estado nutricional regular a ruim e mucosas levemente pálidas. Na abertura da cavidade abdominal evidenciou-se uma massa na face crânio-medial do rim esquerdo (Figura 1).

Essa massa estava aderida a aorta abdominal e as estruturas vasculares adjacentes ao hilo renal (artéria e veia renal). A massa era irregular, macia, branco-avermelhada, com $6,0 \times 4,0 \times 2,0 \mathrm{~cm}$. Ao corte era friável, brancoamarelada com uma cavitação bem delimitada preenchida por conteúdo pastoso enegrecido (Figura 2). 
Figura 1 - Canino, rim, mielolipoma adjacente ao rim esquerdo envolvendo o hilo renal.

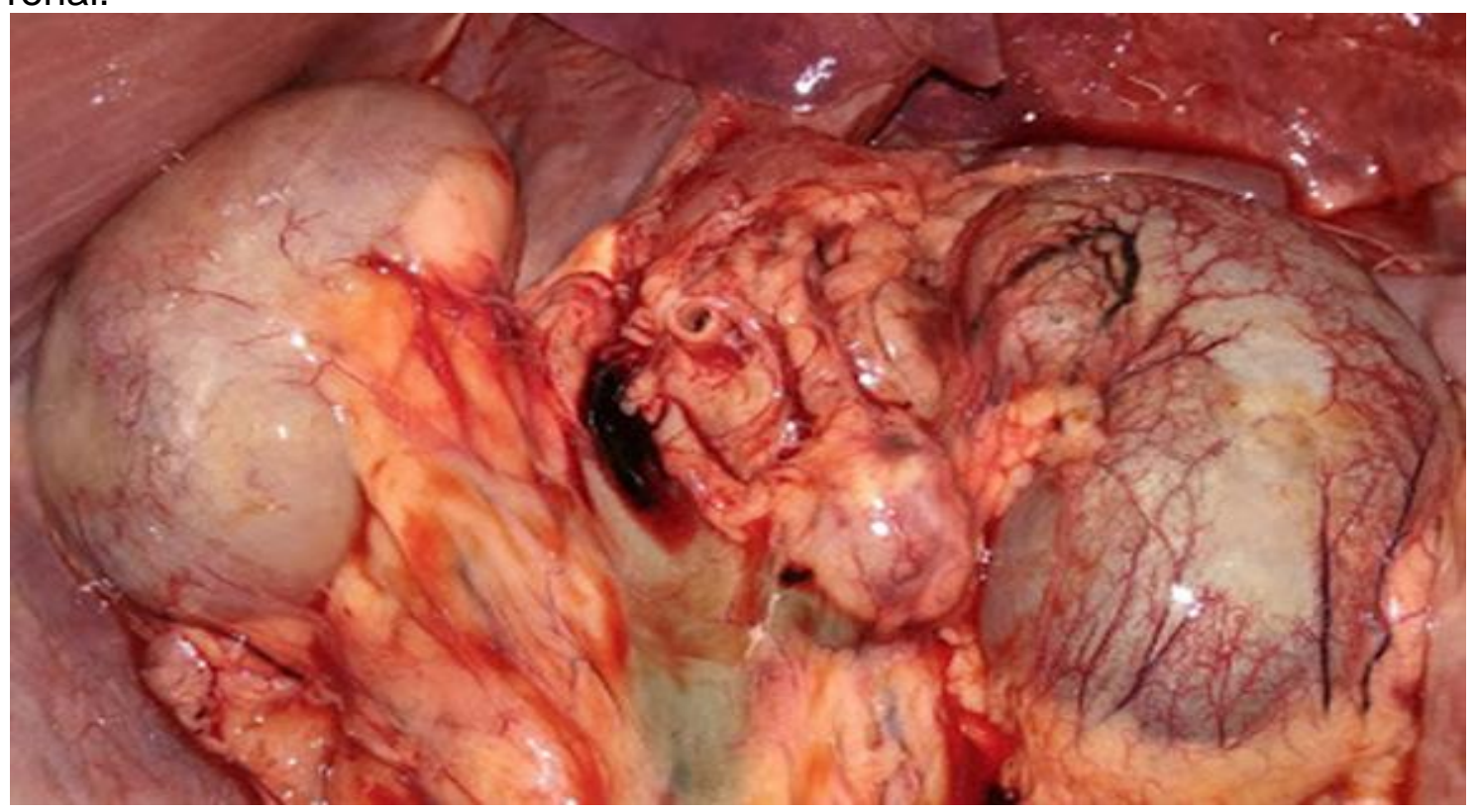

Fonte: os autores.

Figura 2 - Canino, glândula adrenal com massa irregular, branco-amarelada e com cavitações anteriormente preenchidas por conteúdo pastoso, mielolipoma.

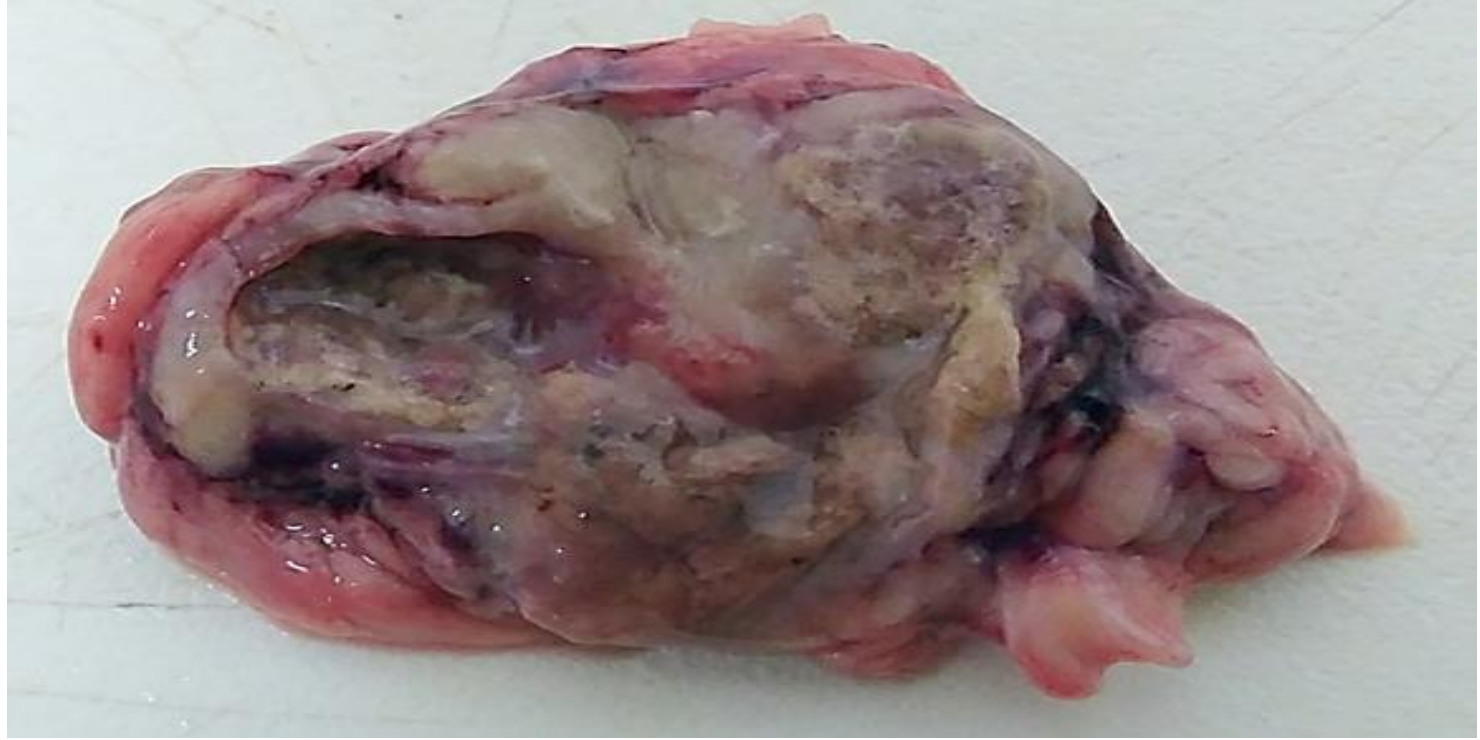

Fonte: os autores.

O rim esquerdo apresentava leve aumento de volume e em sua superfície capsular observou-se congestão moderada. $O$ rim direito apresenta leve redução de tamanho, superfície pálida e irregular com áreas multifocais de depressão e ao corte estava difusamente pálido. A adrenal esquerda não foi evidenciada. Fragmentos dos órgãos foram coletados, fixados em formalina a $10 \%$ e processadas pela técnica rotineira de inclusão em parafina e coloradas pela técnica de Hematoxilina \& Eosina. 
Na histologia da massa notou-se uma capsula acentuadamente espessa e adjacente a esta, poucas células compatíveis com as camadas das zonas glomerulosas, fasciculada e reticulada além de acentuada perda da arquitetura histológica da glândula. Em meio a estas células da porção cortical da glândula adrenal haviam inúmeros adipócitos bem diferenciados circundados por células hematopoiéticas das linhagens mieloide e linfoide (Figura 3 e 4). Haviam áreas multifocais com hemorragia e na porção central, desde a camada medular, se estendendo para a camada cortical da adrenal, notavam-se áreas de necrose associada a presença de trombos vasculares venosos.,

Ambos os rins apresentavam no interstício acentuado infiltrado inflamatório composto por linfócitos, plasmócitos e histiócitos. No córtex renal havia degeneração e necrose do epitélio tubular, sendo esta mais acentuada nos túbulos contorcidos proximais no rim esquerdo. Alguns túbulos estavam preenchidos por debris celulares (cilindros granulosos). Os glomérulos apresentavam moderado espessamento da capsula de Bowman e alguns demostravam redução acentuada do tufo glomerular (esclerose). Na porção medular havia acentuada necrose tubular com deposição de material fortemente basofílico e granular (mineralização). No rim esquerdo havia ainda acentuada presença de eritrócitos preenchendo os capilares venosos (congestão) principalmente na superfície da porção cortical (Figura 5).

Figura 3 - Adrenal, hematoxilina e eosina, obj 10x. Estreitamento da camada granular e rarefação das células granulosas por invasão de adipócitos bem diferenciados circundados por células hematopoiéticas.

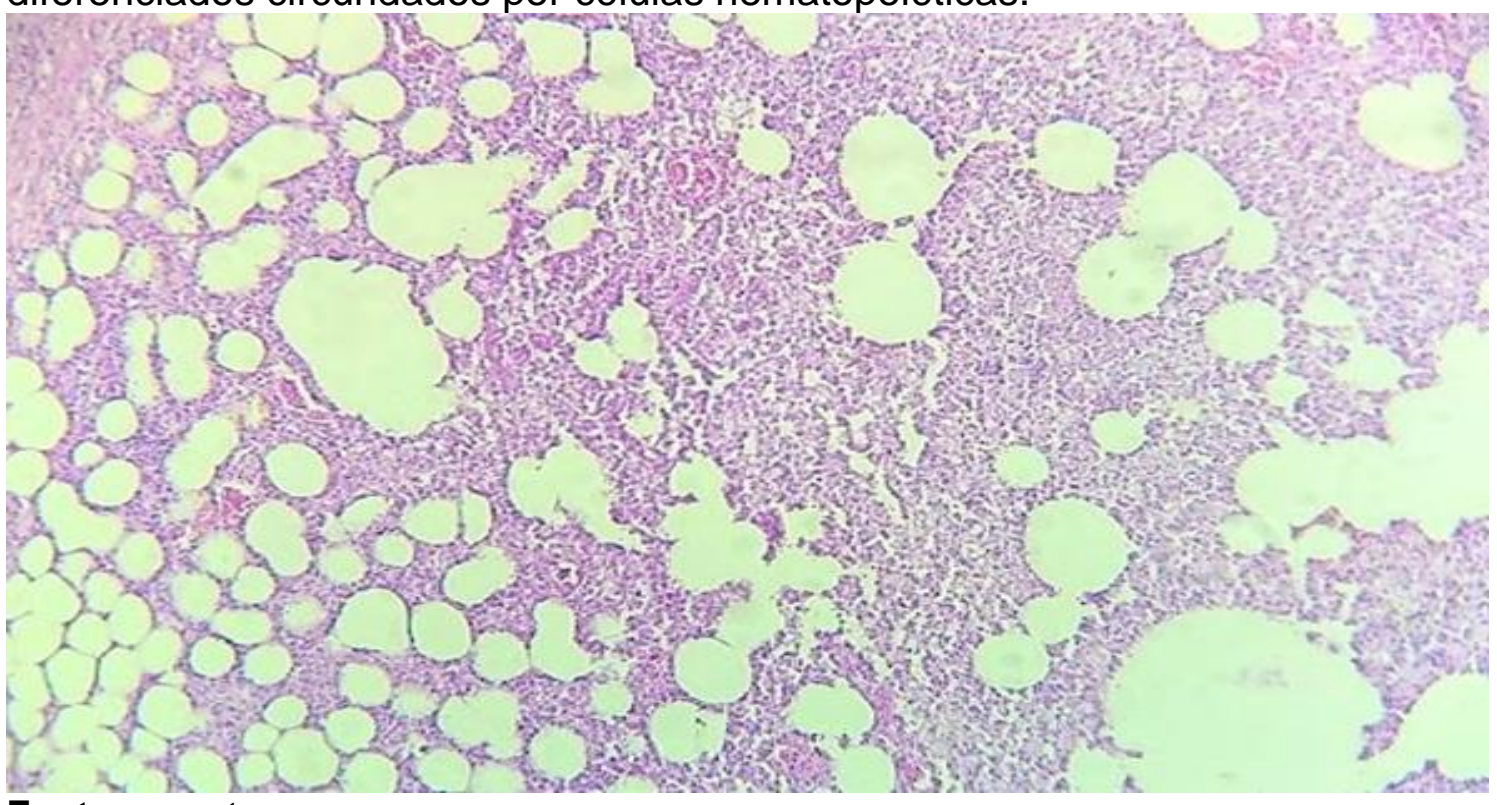

Fonte: os autores. 
Figura 4 - Adrenal, hematoxilina e eosina, objetiva de 40x. Atrofia da camada granular e rarefação das células granulosas por invasão de adipócitos bem diferenciados circundados por células hematopoiéticas.

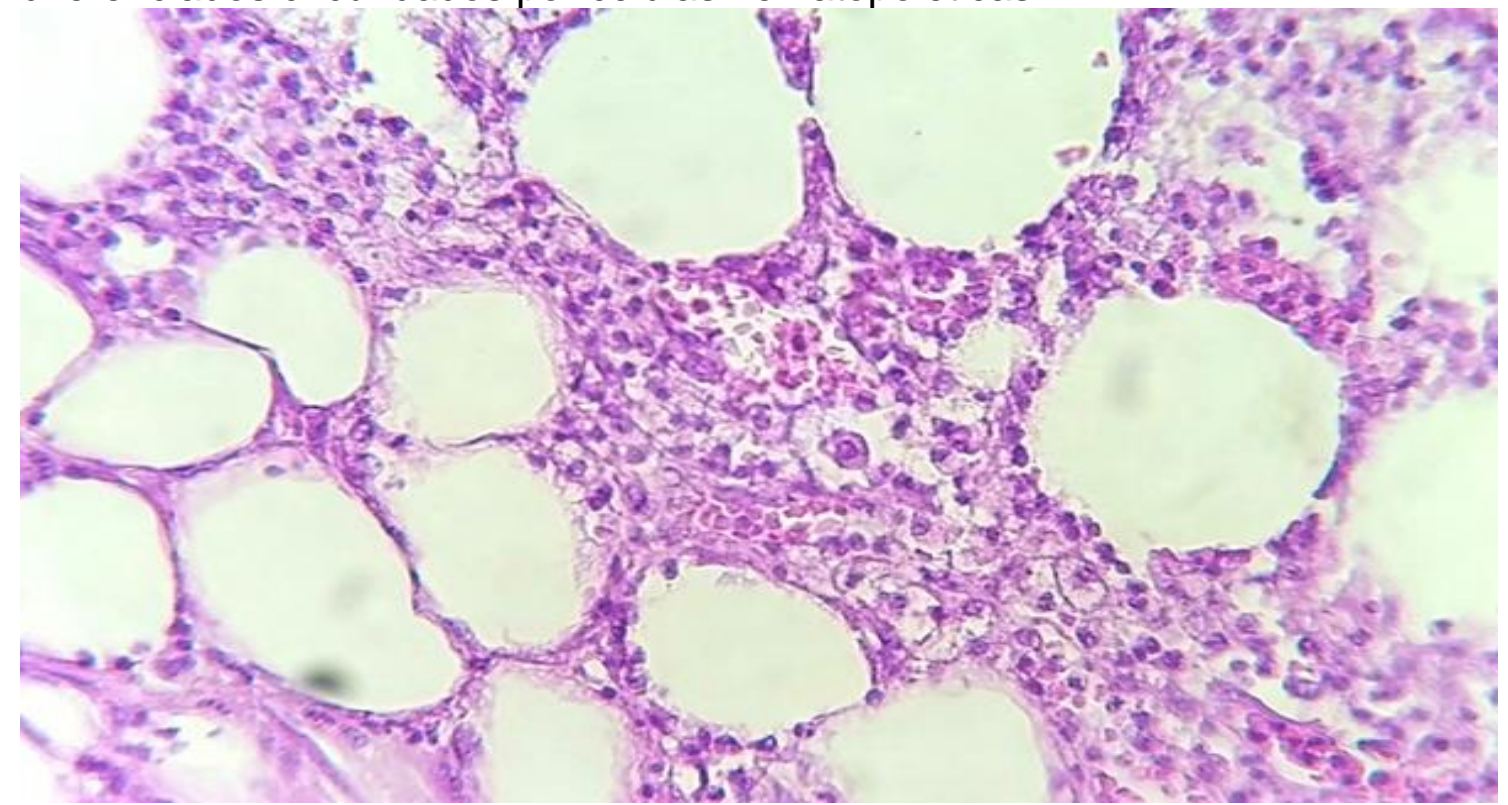

Fonte: os autores.

Figura 5 - Rim esquerdo, hematoxilina e eosina, objetiva de 10x. Infiltrado inflamatório, degeneração e necrose do epitélio tubular, espessamento da capsula de Bowman e marcada presença de eritrócitos preenchendo os capilares venosos (congestão).

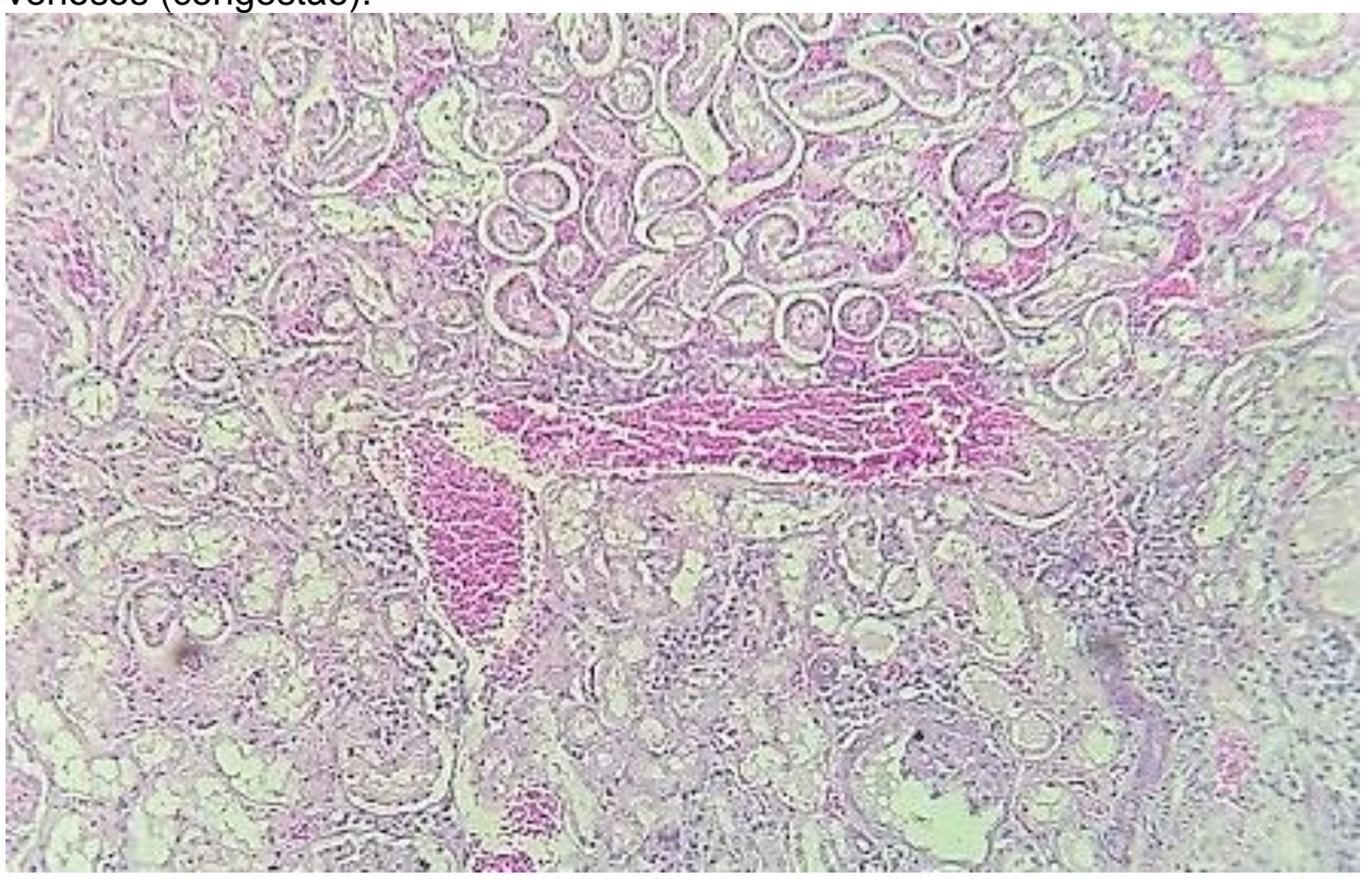

Fonte: os autores. 


\section{DISCUSSÃO}

Diante da análise histopatológica confirmou-se que a massa tratava-se da adrenal esquerda com um neoplasma cuja a descrição histopatológica corrobora com a descrição do mielolipoma (CAPEN, 2002; LABELLE; COCK, 2005; TURSI et al., 2005; FRANÇA; ECCO; GUEDES, 2008; LEDUR et al., 2012; ECCO; LANGOHR, 2016).

Sua etiologia ainda não é completamente esclarecida, porém há diversas teorias que sugerem a origem desse tumor: hiperplasia de adipócitos; embriogênese de adrenal incompleta; hematopoiese extra-medular; ou de um embolismo de medula óssea (COTRAN; KUMAR; ROBBINS, 2000; ECCO; LANGOHR, 2016).

O mielolipoma é uma neoplasia infrequente em cães, sendo pouco relatada nessa espécie (LABELLE; COCK, 2005; TURSI et al., 2005; MORANDI et al., 2007; FRANÇA; ECCO; GUEDES, 2008; LEDUR et al., 2012). É um tumor habitualmente assintomático, sendo encontrado apenas como um achado em exames ultrassonográficos ou necropsia, porém suas complicações baseiam-se na compressão de vísceras adjacentes como fígado, pâncreas e rim (CAPEN, 2002; PATEL et al., 2006).

Além disso, apesar do mielolipoma ser um tumor benigno, Massari et al. (2011) afirma que o tempo de sobrevivência é menor para cães com tumor de glândulas adrenais que apresentem trombose venosa ou massas iguais ou maiores que $5 \mathrm{~cm}$.

No caso em questão, o rim esquerdo apresentava marcada dilatação vascular subcapsular e na análise histopatológica evidenciou-se congestão vascular acentuada. Sabe-se que a congestão local passiva pode decorrer de neoplasias compressivas (JONES; HUNT; KING, 2000; MOSIER, 2012). Dessa forma, acreditamos que devido à extensão do tumor, adjacente ao hilo renal, resultou em compressão na veia renal reduzindo o seu fluxo (estenose venosa).

As outras alterações renais como glomerulonefrite, glomeruloesclerose, necrose tubular difusa moderada e mineralização, encontradas em ambos os rins, não foram relacionadas ao tumor por se tratar de lesões bilaterais, sendo que a massa afetava apenas a porção esquerda.

\section{CONCLUSÃO}

O diagnóstico de mielolipoma de adrenal foi baseado na análise histopatológica e por se tratar de uma neoplasia inativa e não invasiva esta foi considerada um achado de necropsia incidental, sendo a causa da morte deste canino uma doença renal bilateral de origem não determinada.

Apesar deste tumor não estar relacionado ao óbito do animal, foram encontradas lesões compatíveis com compressão venosa no rim esquerdo. Assim, visamos relatar uma neoplasia infrequente em adrenal de cão, bem como alertar clínicos e patologistas veterinários quanto a possibilidade de um tumor de adrenal incomum em cães, como o mielolipoma, ser o causador de lesões por compressão em órgãos e estruturas adjacentes como o rim. 


\section{REFERÊNCIAS}

CAPEN, C. C. Tumors of the endocrine Glands. In: MEUTEN, D.J. Tumors in domestic animals. 4th ed. Ames: lowa State, 2002, p. 509-546.

COTRAN, R. S.; KUMAR, V.; ROBBINS, S. L. Robbins Patologia Estrutural e Funcional. 6th ed. Rio de Janeiro: Guanabara Koogan, p.1268. 2000

ECCO, R.; LANGOHR, I. M. Sistema endócrino. In: SANTOS, R.L.; ALESSI, A.C. Patologia Veterinária. 2nd ed. Rio de Janeiro: Rocap.703-750, 2016;

FRANÇA, S. A.; ECCO, R.; GUEDES, R. M. C. Mielolipoma adrenal bilateral em um cão. Ciência Rural. v. 28, n. 5, p. 1479-1482, 2008.

JONES, T. C.; HUNT, R. D.; KING, N. W. Patologia Veterinária. 6th ed. Barueri: Manole, p. 1415, 2000.

LABELLE, P. et al. Indicators of Malignancy of Canine Adrenocortical Tumors: Histopathology and Proliferation Index. Veterinary Pathology. v. 41, n. 5, p. 490497, 2004.

LABELLE, P.; COCK, H. E. V. Metastatic Tumors to the Adrenal Glands in Domestic Animal. Veterinary Pathology. v. 42, n. 1, p. 52-58, 2005.

LEDUR, G. R. et al. Hemangioma e mielolipoma em glândula adrenal de cão. Acta Scientiae Veterinariae. v. 40, n. 3, p. 1-3, 2012.

MASSARI, F. et al. Adrenalectomy in dogs with adrenal gland tumors: 52 cases (2002-2008). Journal of the American Veterinary Medical Association. v. 239, n. 2, p. 216-221, 2011.

MORANDI, F. et al. Imaging diagnosis bilateral adrenal adenomas and myelolipomas in a dog. Veterinary Radiology \& Ultrasound. v. 48, n. 3, p. 246249, 2007.

MOSIER, D. A. Vascular disorders and thrombosis. In: ZACHARY, J. F., MCGAVIN, M. D. Pathologic basis of veterinary disease. 5th ed. Missouri: Saunders Elsevier, p. 60-88. 2012.

PATEL, V. G. et al. Adrenal myelolipoma: report of a case and review of the literature. The American Surgeon. v. 72, n. 7, p. 649-654, 2006.

TURSI, M. et al. Adrenal myelolipoma in a dog. Veterinary Pathology, v. 42, n. 2, p. 232-235, 2005. 